\title{
The Economical Analysis of the Relationship Between Doctors and Patients Based on Double-Principal-Agent Model
}

\author{
Ying Zhang ${ }^{1, a}$, Zhi Wang ${ }^{2, b}$ \\ ${ }^{1}$ School of Business,Central South University,Changsha 410083,China \\ ${ }^{2}$ School of Business, Central South University, Changsha 410083,China \\ aemail:zhangying9898@sina.com.com; bemail:173901712@qq.com
}

Key word:network platform; double-Principal-Agent model; relationship between doctor and patient; asymmetric information

\begin{abstract}
Along with the development of "Internet plus",the traditional relationship between doctors and patients is undergoing a radical change.Nowadays,it is advisable for patients to search the relevant information about the disease and the doctor through the websites before go to the hospital by themselves. This paper is trying to analyze the new form of relationship between doctors and patients based on the network platform,and draw a safe conclusion that introducing certain identifiable index into the contract can abate the conflict between the doctor and the patient.
\end{abstract}

\section{Introduction}

In recent years, the reports of medical disputes have appeared more frequently. According to a survey conducted by the Chinese Hospital Association ${ }^{[1]}$ in 2013, medical workers were abused and threatened more commonly. To be specifically,the average number of medical disputes per year reached 27.3 in 2012, and the proportion of the attacks on the body of the medical staff had increased by 20 percentage compared with 2011.

Arrow $^{[2]}$ firstly put forward the information asymmetry in the process of medical assistance, using the "Principal-Agent"model to demonstrate the phenomenon.In addition, Thomas Szas ${ }^{[3]}$ had divided the relationship between doctor and patient into three categories:active-passive model;cooperation model; mutual participation model.

However,previous studies had pay little attention to the function of network platform serving between patient/hospital(in this paper,we do not distinguish between hospitals and doctors) and doctor.With the continuous development of the "internet plus", in many cases, patients (especially the special major diseases) will try to acquire information about the target hospital through the network platform before treatment, this way has gradually become a typical practice nowadays.From a positive point of view, patients could master a general understanding of many hospitals within a relatively short time. but at the same time,patients' focus is largely influenced by the propaganda of the network platform,for example:people tend to focus on the objects that are on top of the search results and neglect other objects that on the bottom; people tend to focus on the objects that are repeatedly mentioned but ignore other objects that only mentioned for few times. These habits are largely exploited by hospitals that put tons of money into the network platform to promote themselves, which absolutely damages patients' benefit. Due to the natural differences between the network platform and hospital industry,it is easy to generate moral risk that 
can hardly be eradicated.

Accordingly ,the purpose of this paper is to explain the "patient-network platform-hospital" form.Due to the assumptions that the background is no longer just hospital and patient but add network platform as a medium between them, the paper developed the traditional model into "Double Principal-Agent"model.In this model,There are two principals, namely the hospital and patient, but only one agent, network platform.Firstly, there is one principal-agent relationship between the network platform and the hospital,The hospital serves as the principal by paying advertising fee to the platform to promote itself.In this process, there is a certain degree of beautification and distortion of advertising information.Secondly, there is another principal-agent relationship between the network platform and the patient. Although the patient does not provide the exact payment to platform, but the patient will consume on the network platform through a variety of other ways, such as participation in the network platform for other payment activities, or buy the related peripheral products etc.For the sake of conciseness, this paper equalizes the patients' consumption on the network platform as a contract with network platform, authoring the platform to provide valid information about medical treatment.

\section{Discussion}

For the needs of the discussion, the following assumptions are given:

1 -Assuming that the hospital is the principal A, the patient is the principal $\mathrm{B}$, the network platform is the agent C.The principal and agent's goal is to maximize utility (or maximize revenue).For the convenience of the paper, the output function of the agent network platform is linear: $K_{1}\left(a_{1}\right)=M_{1}+n_{1} a_{1}+\theta_{1}$, The output function of the agent for patient B is $K_{2}\left(a_{2}\right)=M_{2}+n_{2} a_{2}+\theta_{2}, a_{i}(i=1,2), \forall a_{i} \in A$ represents agent's effort,In this paper, the influence of output is assumed to be a one-dimensional variable of the degree of effort, $n_{i}(i=1,2)$ represents contribution rate of labor input to output of network platform. $M_{i}(i=1,2)$ is an exogenous constant. $2 \cdot$ Assuming that the contracts that principal $\mathrm{A}$ and $\mathrm{B}$ provide to the agent network platform are $W_{1}\left(K_{1}\right)=\alpha_{1}+\beta_{1} K_{1}$ and $W_{2}\left(K_{2}\right)=\alpha_{2}+\beta_{2} K_{2}, \alpha_{\mathrm{i}}(i=1,2)$ is regular fee, and $\beta_{\mathrm{i}}(i=1,2)$ is a reward based on output,in exceptional cases, $\beta_{\mathrm{i}}(i=1,2)=0$ means that the agent does not have to take any risks, while $\beta_{\mathrm{i}}(i=1,2)=1$ means that the agent has to take whole risks.

$3 \cdot$ Assuming that the hospital A uses its own unique advantages to occupy the additional rent I from the patient B.Net profit for client $A$ accounted for $\mathrm{t}(0<\mathrm{t}<1)$, therefore, The utility function of the client A and B are $U_{1}=t\left[K_{1}\left(a_{1}\right)-W_{1}\left(K_{1}\right)\right]+I$ and $U_{2}=K_{2}\left(a_{2}\right)-W_{2}\left(K_{2}\right)$ respectively.

4.Assuming that the effort cost of the network platform for A and B can be equivalent to the cost of money,and the cost function of $\mathrm{A}$ and $\mathrm{B}$ are $C_{1}\left(a_{1}, I\right)=b_{1} \frac{\left(a_{1}+I\right)^{2}}{2}$ and $C_{2}\left(a_{2}\right)=b_{2} \frac{a_{2}^{2}}{2}, b_{i}>0(i=1,2)$ is cost coefficient:The greater the $b$, the greater the negative effect of the same effort.The utility functions of the agents are $U_{1}=-e^{-\rho_{1} \omega_{1}}$ and $U_{2}=-e^{-\rho_{2} w_{2}}, \omega_{i}(i=1,2)$ represents real revenue.

\section{Part One}

According to the assumption above,we can calculate that the expected revenue of $\mathrm{A}$ is $E\left(v_{1}\right)=E\left\{t\left[K_{1}\left(a_{1}\right)-W_{1}\left(K_{1}\right)\right]+I\right\}=-\alpha_{1} t+t\left(1-\beta_{1}\right)\left(M_{1}+n_{1} a_{1}\right)+I$,

and the actual revenue of network platform is $\omega_{1}=W_{1}\left(K_{1}\right)-C_{1}\left(a_{1}, I\right)=\alpha_{1}+\beta_{1}\left(M_{1}+n_{1} a_{1}+\theta_{1}\right)-\frac{b_{1}^{2}\left(a_{1}+I\right)^{2}}{2}$,

the Certainty equivalent income of network platform is

$E\left(\omega_{1}\right)-\frac{\rho_{1}\left(t \beta_{1}\right)^{2} \sigma_{1}^{2}}{2}=\alpha_{1}+\beta_{1}\left(M_{1}+n_{1} a_{1}\right)-\frac{1}{2} b_{1}\left(a_{1}+I\right)^{2}-\frac{1}{2} \rho_{1}\left(t \beta_{1}\right)^{2} \sigma_{1}^{2}$,

In the equation above, $E\left(w_{1}\right)$ represents the expected revenue of $C, \frac{1}{2} \rho_{1}\left(t \beta_{1}\right)^{2} \sigma_{1}^{2}$ represents the risk cost of $C$,especially,when $\beta_{1}=0$, the risk cost of $C$ is zero.

According to "state-space formulation" ${ }^{[4]}$, the target of $\mathrm{A}$ is to choose suitable $a_{1}^{*}$ and $W_{1}^{*}\left(K_{1}\right)$ to 
maximize the expected revenue:

$\operatorname{Max} E\left(v_{1}\right)=E\left\{t\left[K_{1}\left(a_{1}\right)-W_{1}\left(K_{1}\right)\right]+I\right\}=-\alpha_{1} t+t\left(1-\beta_{1}\right)\left(M_{1}+n_{1} a_{1}\right)+I$

S.t. $\quad(\mathrm{IR}) \quad E\left[W_{1}\left(K_{1}\right)-C_{1}\left(a_{1}, I\right)\right]-\frac{1}{2} \rho_{1}\left(t \beta_{1}\right)^{2} \sigma_{1}^{2} \geq 0$

(IC)

$$
\operatorname{Max} E\left[W_{1}\left(K_{1}\right)-C_{1}\left(a_{1}^{*}, I\right)\right]-\frac{1}{2} \rho_{1}\left(t \beta_{1}\right)^{2} \sigma_{1}^{2}
$$

IR is the abbreviation of individual rational constraint,and it means that the minimum expected return from the contract shall not be less than the maximum expected return from rejecting the contract; IC is the abbreviation of incentive compatibility constraint,and it means that the contract must lead him to choose voluntarily to meet the maximum utility.

Due to the the first-order approach, we can calculate from(3)that:

$\frac{\partial E\left(\omega_{1}\right)}{\partial a_{1}}=0$, which means $a_{1}^{*}=\frac{\beta_{1} n_{1}}{b_{1}}-I$

So the original problem can be rewritten as

$\operatorname{Max} t\left[M_{1}+n_{1}\left(\frac{\beta_{1} n_{1}}{b_{1}}-I\right)\right]-\frac{t \beta_{1}{ }^{2} n_{1}^{2}}{2 b_{1}}-\frac{t}{2} \rho_{1}\left(t \beta_{1}\right)^{2} \sigma_{1}{ }^{2}+I$,

First order condition is : $\frac{t n_{1}^{2}}{b_{1}}-\frac{n_{1}^{2} \beta_{1} t}{b_{1}}-\rho_{1} \beta_{1} t^{2} \sigma_{1}^{2}=0$,

So $\beta_{1}^{*}=\frac{n_{1}^{2}}{n_{1}^{2}+t b_{1} \rho_{1} \sigma_{1}^{2}}, a_{1}^{*}=\frac{n_{1}^{3}}{\left(n_{1}^{2}+t b_{1} \rho_{1} \sigma_{1}{ }^{2}\right) b}-I$,

The expected utility of $\mathrm{A}$ is

$E\left(U_{1}^{*}\right)=-\alpha_{1} t+t\left(1-\frac{n_{1}^{2}}{n_{1}^{2}+t b_{1} \rho_{1} \sigma_{1}^{2}}\right)\left[M_{1}+\frac{n_{1}^{4}}{\left(n_{1}^{2}+t b_{1} \rho_{1} \sigma_{1}^{2}\right) b}\right]+I$

Expected output generated by network platform is

$$
E\left(K_{1}^{*}\right)=M_{1}+n_{1}\left(\frac{n_{1}^{3}}{n_{1}^{2} b+t \rho_{1} b_{1}^{2} \sigma_{1}^{2}}-I\right)
$$

the Certainty equivalent income of network platform is

$$
\alpha_{1}+\frac{n_{1}{ }^{2}}{n_{1}{ }^{2}+t b_{1} \rho_{1} \sigma_{1}{ }^{2}}\left[M_{1}+n_{1}\left(\frac{n_{1}{ }^{2}}{n_{1}{ }^{2} b_{1}+t b_{1}{ }^{2} \rho_{1} \sigma_{1}{ }^{2}}-I\right)\right]-\frac{\left(n_{1} \beta_{1}\right)^{2}}{2 b_{1}}-\frac{1}{2} \rho_{1}\left(\frac{n_{1}{ }^{2} t}{n_{1}{ }^{2}+t b_{1} \rho_{1} \sigma_{1}{ }^{2}}\right)^{2} \sigma_{1}{ }^{2}
$$

\section{Part Two}

According to "state-space formulation", the target of B is to choose suitable $a_{2}^{*}$ and $W_{2}^{*}\left(K_{2}\right)$ to maximize the expected revenue:

$\operatorname{Max} E\left(v_{2}\right)=E\left[K_{2}\left(a_{2}\right)-W_{2}\left(K_{2}\right)\right]=-\alpha_{2}+\left(1-\beta_{2}\right)\left(M+n_{2} a_{2}\right)$

S.t. (IR) $E\left[W_{2}\left(K_{2}\right)-C_{2}\left(a_{2}\right)\right]-\frac{1}{2} \rho_{2} \beta_{2}^{2} \sigma_{2}^{2} \geq 0$

$$
\text { (IC)Max } E\left[W_{2}\left(K_{2}\right)-C_{2}\left(a_{2}^{*}\right)\right]-\frac{1}{2} \rho_{2} \beta_{2}{ }_{2} \sigma_{2}^{2}
$$

Due to the the first-order approach, we can calculate from(10)that:

$$
\frac{\partial E\left(\omega_{2}\right)}{\partial a_{2}}=0, \text { which means } a_{2}=\frac{\beta_{2} n_{2}}{b_{2}}
$$

So the original problem can be rewritten as

$\operatorname{Max}\left(M_{2}+\frac{\beta_{2} n_{2}^{2}}{b_{2}}\right)-\frac{1}{2} \rho_{2} \beta_{2}^{2} \sigma_{2}^{2}-\frac{b_{2}}{2}\left(\frac{\beta_{2} n_{2}}{b_{2}}\right)^{2}$,

So $\beta_{2}^{*}=\frac{n_{2}^{2}}{n_{2}^{2}+b_{2} \rho_{2} \sigma_{2}^{2}}, a_{2}^{*}=\frac{n_{2}{ }^{3}}{b_{2} n_{2}^{2}+\rho_{2} b_{2}{ }^{2} \sigma_{2}^{2}}$ 
Expected output generated by network platform is

$$
E\left(K_{2}^{*}\right)=M_{2}+\frac{n_{2}^{4}}{b_{2} n_{2}^{2}+\rho b_{2}^{2} \sigma_{2}^{2}}
$$

\section{Part Three}

In order to solve the information asymmetry between doctors and patients, previous studies contended that making doctors' information publicity is an effective way, however,in the background of "internet plus", attention can be focused on the network platform,since it is more easier to be monitored and regulated than the individual patient or doctor.In the discussion below, we will examine the effectiveness of adding an observable parameter.

For the conciseness of the discussion,we assumed that $\mathrm{Z}$ is a variable that can be observed by the national Network Technology Department, and has nothing to do with the level of effort, but is related to the exogenous variables, which is related to the output $K$. Meanwhile, $E(z)=0 \quad, \operatorname{Var}(z)=\sigma_{z}^{2}$. So, the contract between patient and network platform is $W_{2}\left(K_{2}, z\right)=\alpha_{2}+\beta_{2}\left(K_{2}+\gamma z\right)$, in this new background, the actual revenue of network platform is $\alpha_{2}+\beta_{2}\left(M_{2}+n_{2} a_{2}\right)-\frac{1}{2} b_{2} a_{2}{ }^{2}-\frac{1}{2} \rho_{2} \beta_{2}{ }^{2}\left[\sigma_{2}^{2}+\gamma^{2} \sigma_{z}^{2}+2 \gamma \operatorname{cov}\left(K_{2}, \mathrm{z}\right)\right]$ , $\operatorname{cov}\left(K_{2}, \mathrm{z}\right)$ represents covariation.

The expected revenue of B is $E\left[K_{2}-\alpha_{2}-\beta_{2}\left(K_{2}+\gamma z\right)\right]=-\alpha_{2}+\left(1-\beta_{2}\right)\left(M_{2}+n_{2} a_{2}\right)$ So ,the original problem can be rewritten as $\operatorname{Max}\left(M_{2}+\frac{\beta_{2} n_{2}{ }^{2}}{b_{2}}\right)-\frac{1}{2 b_{2}}\left(\beta_{2} n_{2}\right)^{2}-\frac{1}{2} \rho_{2} \beta_{2}{ }^{2}\left[\sigma_{2}{ }^{2}+\gamma^{2} \sigma_{z}^{2}+2 \gamma \operatorname{cov}\left(K_{2}, z\right)\right]-\overline{w_{2}}$, So $\beta_{2}^{\prime}=\frac{n_{2}^{2}}{n_{2}^{2}+b_{2} \rho_{2}\left(\sigma_{2}^{2}-\operatorname{cov}^{2}\left(K_{2}, z\right) / \sigma_{z}^{2}\right)}, \quad \gamma=-\frac{\operatorname{cov}\left(K_{2}, z\right)}{\sigma_{z}^{2}}$

\section{Result}

$1 \cdot \operatorname{according}$ to $(6), \frac{\partial E\left(K_{1}^{*}\right)}{\partial M_{1}}>0, \frac{\partial E\left(K_{1}^{*}\right)}{\partial n_{1}}>0, \frac{\partial E\left(K_{1}^{*}\right)}{\partial t}>0$,

which means when the influence of the network platform is wider, the expected income of the network platform will be higher, and the proportion of the income of the client will also increase. $2 \cdot \operatorname{according}$ to $(6), \frac{\partial E\left(K_{1}^{*}\right)}{\partial \rho_{1}}<0, \frac{\partial E\left(K_{1}^{*}\right)}{\partial b_{1}}<0, \frac{\partial E\left(K_{1}^{*}\right)}{\partial I}<0$, which means when the cost coefficient of network platform increases, the occupation of the patient's principal's rent will increase.

$3 \cdot \operatorname{according}$ to $(12), \frac{\partial E\left(K_{2}^{*}\right)}{\partial b_{2}}<0, \frac{\partial E\left(K_{2}^{*}\right)}{\partial \rho_{2}}<0$,

which means when the cost coefficient of network platform increases, the expected revenue of network platform will reduce.

4 according to (11)and(13), $\beta_{2}^{\prime}>\beta_{2}$, which means in the improved model, the incentive system is improved, and $\operatorname{Var}\left(W_{2}\left(K_{2}, \mathrm{z}\right)\right)=\beta_{2}{ }_{2}^{2}\left[\sigma_{2}{ }^{2}+\gamma^{2} \sigma_{z}{ }^{2}+2 \gamma \operatorname{cov}\left(K_{2}, z\right)\right]<\operatorname{Var}\left(W_{2}\left(K_{2}\right)\right)$, which Indicates that the risk of network platform will decline, and the risk cost of network platform will reduce too. 


\section{Conclusion}

1 for hospital/doctor, first of all, the hospital should choose a network platform with a good reputation and wide influence to release information, and it can motivate platform's efforts by improving the incentive of the contract,and therefore improve its own expected revenue.In addition, when the revenue of the network platform maximizes, the expected income of the network platform is positively correlated with the income of the hospital, but it has an inverse correlation with the rent I,which would lead to a "lemon market".

2 - for patients,first of all,patients should choose a network platform with a good reputation and wide influence to consult potential hospital information, and patients can also motivate platform's efforts and reduce the cost coefficient by improving the incentive of the contract.

3 - for relevant administration section, as long as we can add an indicator that can be observed by relevant administration section, the agency cost will absolutely reduce and eliminate the information asymmetry between doctors and patients to some degree,the premise here is that the cost of acquiring new indicators is small or even negligible.

\section{References}

[1]Information on http://news.medlive.cn/all/info-news/show-57375_97.html

[2]Kenneth J.Arrow.Uncertainty and the Welfare Economics of Medical Care,edited by The American Economic Review,Vol.53,No.5(Dec,1963):941-973

[3]Thomas Szasz:The myth of mental illness(Trans Harper\&Row Press,America2001)

[4]Eric Rasmusan.Games and Information(Trans China Renmin University Press, China 2009)

[5]Holmstrom,B.Moral Hazard and Observability,Bell Journal of Economics, 1979(10):74-91

[6]Holmstrom,Milgrom.Aggregation and Linearity in the Provision of Intertemporal Incentives. edited by Econometrica,1987(55):303-328

[7]Holmstrom,Milgrom.Multi-Task Principal-Task Analyses:Incentive Contracts, Asset Ownership and Job Design.edited by Econometrics and Organization.1991(7):24-52

[8]Holmstrom,Ricard I Costa.Managerial Incentives and Capital Management.edited by Quarterly Journal of Economics.1986:403-460

[9]Itoh.Incentives for Help in Multi-Agency Situation,edited by Econometrica. 1991:611-636 